\title{
HEAT INVARIANTS OF RIEMANNIAN MANIFOLDS
}

\author{
IOSIF POLTEROVICH
}

\begin{abstract}
We calculate heat invariants of arbitrary Riemannian manifolds without boundary. Every heat invariant is expressed in terms of powers of the Laplacian and the distance function. Our approach is based on a multi-dimensional generalization of the Agmon-Kannai method. An application to computation of the Korteweg-de Vries hierarchy is also presented.
\end{abstract}

\section{IntRodUCtION AND MAIN RESULtS}

1.1. Heat invariants. Let $M$ be a $d$-dimensional Riemannian manifold without boundary with a metric $\left(g_{i j}\right)$, and $\Delta$ be the LaplaceBeltrami operator (or simply the Laplacian) on $M$. In local coordinates $\left(x_{1}, \ldots, x_{d}\right)$ the Laplacian is given by

$$
\Delta f=-\frac{1}{\sqrt{g}} \sum_{i, j=1}^{d} \frac{\partial\left(\sqrt{g} g^{i j}\left(\partial f / \partial x_{i}\right)\right)}{\partial x^{j}},
$$

where $g=\operatorname{det}\left(g_{i j}\right)$, and $\left(g^{i j}\right)$ denotes the inverse of the matrix $\left(g_{i j}\right)$.

The heat kernel $K(t, x, y)$ is the fundamental solution of the heat equation

$$
\left(\frac{\partial}{\partial t}+\Delta\right) f=0 .
$$

The function $K(t, x, y)$ is analytic in $t>0$ and $C^{\infty}$ in $x$ and $y$, and has the following asymptotic expansion on the diagonal as $t \rightarrow 0+$ (see [G1]):

$$
\mathrm{K}(t, x, x) \sim \sum_{n=0}^{\infty} a_{n}(x) t^{n-\frac{d}{2}}
$$

It is called the Minakshisundaram-Plejel asymptotic expansion ([MP]). The coefficients $a_{n}(x)$ are (local) heat invariants of the manifold $M$. They are homogeneous polynomials of degree $2 n$ in the derivatives of the Riemannian metric $\left\{g^{i j}\right\}$ at the point $x$ (see [G2]). Integrating $a_{n}(x)$ over the manifold one gets the coefficients $a_{n}$ of the expansion 
for the trace of the heat operator $e^{-t \Delta}$ :

$$
\sum_{i} e^{-t \lambda_{i}} \sim \sum_{n=0}^{\infty}\left(\int_{M} a_{n}(x) \sqrt{g} d x\right) t^{n-\frac{d}{2}} \sim \sum_{n=0}^{\infty} a_{n} t^{n-\frac{d}{2}} .
$$

Computation of heat invariants is a well-known problem in spectral geometry (see [Be], [G1], [Ch], [G3], [Ro]) which has various applications $([\mathrm{F}],[\mathrm{P} 2])$. The first method for derivation of heat kernel asymptotics is due to Seeley ([Se]). This method was deveoped later by Gilkey (see Theorem 1.3 in [G1]) who presented a way to get recursive formulas for the heat invariants.

However, explicit formulas for $a_{n}(x)$ in arbitrary dimension existed only for $n \leq 5$ ([MS], [Sa], $[\mathrm{Av}],[\mathrm{vdV}])$. The reason for this is the combinatorial complexity of $a_{n}(x)$ which is increasing very rapidly with the growth of $n$. For the higher heat invariants only partial information is known $([\mathrm{BG} \varnothing],[\mathrm{OPS}])$. Let us also mention interesting recursive formulas for $a_{n}(x)$ obtained in [Xu].

In this paper we calculate all heat invariants $a_{n}(x)$ for an arbitrary Riemannian manifold without boundary in terms of powers of the Laplacian and the distance function. Note that the heat invariants were initially given by a recursive system of differential equations involving exactly the Laplacian and the distance function (see $[\mathrm{MP}]$ ).

1.2. Main result. Given a point $x \in M$ denote by $\rho_{x}: M \rightarrow \mathbb{R}$ the corresponding distance function: for every $y \in M$ the distance between the points $y$ and $x$ is $\rho_{x}(y)$.

Theorem 1.2.1. Heat invariants $a_{n}(x)$ are equal to

$$
a_{n}(x)=\left.(4 \pi)^{-d / 2}(-1)^{n} \sum_{j=0}^{3 n}\left(\begin{array}{c}
3 n+\frac{d}{2} \\
j+\frac{d}{2}
\end{array}\right) \frac{1}{4^{j} j !(j+n) !} \Delta^{j+n}\left(\rho_{x}(y)^{2 j}\right)\right|_{y=x} .
$$

The binomial coefficients for $d$ odd are defined by (4.1.2).

1.3. Structure of the paper. In [P1], [P2] we have developed a method for computation of heat invariants based on the Agmon-Kannai asymptotic expansion of resolvent kernels of elliptic operators $([\mathrm{AK}])$. In $[\mathrm{P} 1]$ this method is used to obtain explicit formulas for the heat invariants of 2-dimensional Riemannian mainfolds, in [P2] - for computation of the Korteweg-de Vries (KdV) hierarchy via heat kernel coefficients of the 1-dimensional Schrödinger operator. In this paper we present a multi-dimensional generalization of the Agmon-Kannai method which is described in section 2.3. In section 3.1 we apply it to get formulas for the heat invariants in normal coordinates. It turns out that combinatorial coefficients in these formulas can be substantially 
simplified which is done in section 4.1. In section 4.2 we present $a_{n}(x)$ in a completely invariant form and prove Theorem 1.2.1. Main result allows to simplify the formulas for the KdV hierarchy obtained in [P2]. This is shown in sections 5.1 and 5.2.

\section{Asymptotics of DeRivatives of the RESOlvent}

2.1. A modification of Agmon-Kannai expansion. The original Agmon-Kannai theorem ([AK] $)$ deals with asymptotic behaviour of resolvent kernels of elliptic operators. In [P1] we have obtained a concise reformulation of this theorem which is suitable for computation of heat invariants. We start with some notations.

Let $H$ be a a self-adjoint elliptic differential operator of order $p$ on a Riemannian manifold $\left(M, g_{i j}\right)$ of dimension $d<p$ and let $H_{0}$ be the operator obtained by freezing the coefficients of the principal part $H^{\prime}$ of the operator $H$ at some point $x \in M: H_{0}=H^{\prime}(x)$. Denote by $R_{\lambda}(x, y)$ the kernel of the resolvent $R_{\lambda}=(H-\lambda)^{-1}$, and by $F_{\lambda}(x, y)$ — the kernel of $F_{\lambda}=\left(H_{0}-\lambda\right)^{-1}$.

Theorem 2.1.1. ([P1]). The resolvent kernel $R_{\lambda}(x, y)$ has the following asymptotic representation on the diagonal as $\lambda \rightarrow \infty$ :

$$
R_{\lambda}(x, x) \sim \frac{1}{\sqrt{g}} \sum_{m=0}^{\infty} X_{m} F_{\lambda}^{m+1}(x, x),
$$

where the operators $X_{m}$ are defined by:

$$
X_{m}=\sum_{k=0}^{m}(-1)^{k}\left(\begin{array}{c}
m \\
k
\end{array}\right) H^{k} H_{0}^{m-k}, m \geq 0 .
$$

2.2. Derivatives of the resolvent. The main obstruction for using Theorem 2.1.1 directly for computation of heat invariants of a $d$ dimensional Riemannian manifold is the condition $d<p$, where $p=2$ is the order of the Laplacian. In [P1] we avoid this difficulty for 2dimensional manifolds taking the difference of resolvents. However, in the general case one should consider derivatives of the resolvent kernel (cf. $[\mathrm{AvB}])$.

Lemma 2.2.1. The following asymptotic expansion on the diagonal holds for the derivatives of the resolvent kernel of the Laplacian on a $d$-dimensional Riemannian manifold $M$ :

$$
\frac{d^{s}}{d \lambda^{s}} R_{\lambda}(x, x) \sim \sum_{n=0}^{\infty} \Gamma\left(s+n-\frac{d}{2}+1\right) a_{n}(x)(-\lambda)^{\frac{d}{2}-s-n-1}, \quad s \geq d / 2,
$$

where $a_{n}(x)$ are heat invariants of the manifold $M$. 
Proof. Let $\operatorname{Re} \lambda<0$. We have (formally):

$$
\int_{0}^{\infty} e^{-t(\Delta-\lambda)} d t=\frac{1}{\Delta-\lambda}
$$

Differentiating $R_{\lambda} s$ times with respect to $\lambda$ we get a self-adjoint operator from $L^{2}(M)$ into the Sobolev space $\mathrm{H}^{2 s+2}(M)$. Since $2 s+2>\operatorname{dim} M$ this operator has a continuous kernel (see $[\mathrm{AK}]$ ). Taking into account (1.1.2) we formally have:

$$
\frac{d^{s}}{d \lambda^{s}}\left(\frac{1}{\Delta-\lambda}\right)=\int_{0}^{\infty} t^{s} e^{-t(\Delta-\lambda)} d t \sim \sum_{n=0}^{\infty} a_{n} \int_{0}^{\infty} t^{s+n-d / 2} e^{\lambda t} d t
$$

The asymptotic expansion in (2.2.3) is obviously valid if we integrate over a finite interval $[0, T]$. In order to show that it remains true in our case as well we need an additional argument.

Indeed, it is well-known (for example, see [Da]) that

$$
\left|e^{-t \Delta}\right| \leq c t^{-d / 2}
$$

Therefore we have:

$$
\left|\int_{0}^{\infty} t^{s} e^{-t(\Delta-\lambda)} d t-\int_{0}^{T} t^{s} e^{-t(\Delta-\lambda)} d t\right| \leq \int_{T}^{\infty} c t^{s-d / 2} e^{\lambda t} d t
$$

Let us estimate the second integral. We have:

$$
\int_{T}^{\infty} t^{s-d / 2} e^{\lambda t} d t \leq e^{-\varepsilon T} \int_{T}^{\infty} t^{s-d / 2} e^{(\lambda+\varepsilon) t} d t \leq e^{-\varepsilon T} \frac{\Gamma(s-d / 2+1)}{(\lambda+\varepsilon)^{s-d / 2+1}}
$$

Take $\varepsilon=\sqrt{|\lambda|}$. Then for $T=1$ this is $O\left(e^{-\sqrt{|\lambda|}}\right)$ and therefore the term

$$
\int_{T}^{\infty} t^{s-d / 2} e^{\lambda t} d t
$$

is negligent. This proves the asymptotic expansion in (2.2.3).

The right-hand side of $(2.2 .3)$ is equal to

$$
\sum_{n=0}^{\infty} \frac{a_{n}}{(-\lambda)^{s+n+1-d / 2}} \int_{0}^{\infty} u^{s+n-d / 2} e^{-u} d u=\sum_{n=0}^{\infty} \frac{\Gamma(s+n+1-d / 2) a_{n}}{(-\lambda)^{s+n+1-d / 2}}
$$

and this completes the proof of the lemma. 


\subsection{Agmon-Kannai expansion for derivatives of the resolvent.} In the notations of Theorem 2.1.1 let $H=\Delta$ be the Laplacian on a $d$ dimensional Riemannian manifold $M$, and $\Delta_{0}$ be the operator obtained from the principal part of the Laplacian by freezing its coefficients at a certain point $x \in M$. As before, $R_{\lambda}=(\Delta-\lambda)^{-1}, F_{\lambda}=\left(\Delta_{0}-\lambda\right)^{-1}$.

Theorem 2.3.1. The following asymptotic expansion on the diagonal holds for the derivatives of the resolvent kernel of the Laplacian on a $d$-dimensional Riemannian manifold $M$ :

$$
\frac{d^{s}}{d \lambda^{s}} R_{\lambda}(x, x) \sim \frac{1}{\sqrt{g}} \sum_{m=0}^{\infty} \frac{(m+s) !}{m !} X_{m} F_{\lambda}^{m+s+1}, \quad s \geq d / 2 .
$$

Proof. Formally we have:

$$
\frac{d}{d \lambda} F_{\lambda}=\frac{d}{d \lambda}\left(\frac{1}{\Delta_{0}-\lambda}\right)=\frac{1}{\left(\Delta_{0}-\lambda\right)^{2}}=F_{\lambda}^{2} .
$$

This implies

$$
\frac{d^{s}}{d \lambda^{s}} F_{\lambda}^{m+1}=\frac{(m+s) !}{m !} F_{\lambda}^{m+s+1} .
$$

Together with (2.1.2) this completes the proof of the theorem.

Let us introduce the standard multi-index notations (see [Hö]): if $\alpha=\left(\alpha_{1}, \ldots, \alpha_{d}\right)$ is a multi-index, then $|\alpha|=\alpha_{1}+\cdots+\alpha_{d}, \alpha !=$ $\alpha_{1} ! \cdots \alpha_{d}$ !. For any vector $x=\left(x_{1}, \ldots, x_{d}\right)$ we denote $x^{\alpha}=x_{1}^{\alpha_{1}} \cdots x_{d}^{\alpha_{d}}$ and

$$
\frac{\partial^{\alpha}}{\partial x^{\alpha}}=\frac{\partial^{\alpha_{1}}}{\partial x_{1}^{\alpha_{1}}} \cdots \frac{\partial^{\alpha_{d}}}{\partial x_{d}^{\alpha_{d}}}
$$

We note that (2.3.2) and (2.1.2) are in fact asymptotic expansions in the powers of $-\lambda$ as well as (2.2.2). This is due to the following formula (see $[\mathrm{AK}])$ :

$$
\begin{aligned}
& \frac{\partial^{\gamma}}{\partial x^{\gamma}} F_{\lambda}^{m+s+1}(x, x)= \\
&(-\lambda)^{\frac{d+|\gamma|}{2}-m-s-1} \frac{(-1)^{\frac{|\gamma|}{2}}}{(2 \pi)^{d}} \int_{\mathbb{R}} \frac{\xi^{\gamma} d \xi}{\left(\Delta_{0}(\xi)^{2}+1\right)^{m+s+1}},
\end{aligned}
$$

where $\Delta_{0}(\xi)$ denotes the symbol of the operator $\Delta_{0}, \gamma=\left(\gamma_{1}, \ldots, \gamma_{d}\right)$ is a multi-index and $\xi=\left(\xi_{1}, \ldots, \xi_{d}\right)$.

\section{Heat invariants in nORMal COORDinates}

3.1. Computation of heat invariants. Let $\left(x_{1}, \ldots, x_{d}\right)$ be local coordinates on the Riemannian manifold $M$ such that the Riemannian metric at the origin $x=(0, \ldots, 0) \in M$ (in the sequel we simply write 
$x=0$ ), is Euclidean: $\left.g_{i j}\right|_{x=0}=\delta_{i j}$ For convenience we may consider normal coordinates on $M$ centered at the point $x=0$ (see [GKM]).

Theorem 3.1.1. Let $M$ be a d-dimensional Riemannian manifold without boundary and $\left(x_{1}, \ldots, x_{d}\right)$ be normal coordinates on $M$ centered at the point $x=0$. Then the heat invariants $a_{n}(x)$ at the point $x=0$ are equal to:

$$
\begin{aligned}
a_{n}(0)=(4 \pi)^{-\frac{d}{2}}(-1)^{n} \sum_{m=n}^{4 n} \sum_{k=n}^{m} \frac{1}{k ! 2^{2 m-2 n}} . \\
\left.\cdot \sum_{|\alpha|=m-k} \sum_{|\beta|=k-n} \frac{(2 \alpha+2 \beta) !}{\alpha !(\alpha+\beta) !(2 \beta) !} \Delta^{k}\left(x^{2 \beta}\right)\right|_{x=0},
\end{aligned}
$$

where $\alpha=\left(\alpha_{1}, \ldots, \alpha_{d}\right), \beta=\left(\beta_{1}, \ldots, \beta_{d}\right)$ are multi-indices.

Proof of Theorem 3.1.1 Since $\left(x_{1}, \ldots, x_{d}\right)$ are normal coordinates centered at $x=0$, the principal part of the Laplacian at this point coincides with the Euclidean Laplacian, i.e.:

$$
\Delta_{0}=-\frac{\partial^{2}}{\partial x_{1}^{2}}-\cdots-\frac{\partial^{2}}{\partial x_{d}^{2}}
$$

Due to (2.2.2), in order to compute the coefficient $a_{n}(x)$ we have to collect all terms in the expansion (2.3.2) containing $(-\lambda)^{d / 2-s-n-1}$. From (2.3.3) we have:

$$
\frac{d+|\gamma|}{2}-m-s-1=\frac{d}{2}-s-n-1,
$$

which implies $|\gamma|=2 m-2 n$ and in particular $m \geq n$. As it was shown in [P1], estimates on the orders of operators $X_{m}$ (namely, Lemma 3.1 and Theorem 5.1 in $[\mathrm{AK}])$ imply that $m \leq 4 n$.

Note that due to (3.1.3 all indices $\gamma_{1}, \ldots, \gamma_{2}$ should be even since otherwise the integral in (2.3.3) will vanish. Setting $\gamma=2 \mu=\left(2 \mu_{1}, \ldots, 2 \mu_{d}\right)$ and taking into account that $|\mu|=m-n$ we compute this integral (see [GR]):

$$
\int_{\mathbb{R}} \frac{\xi^{2 \mu} d \xi}{\left(\xi^{2}+1\right)^{m+s+1}}=\frac{\Gamma\left(\mu_{1}+\frac{1}{2}\right) \Gamma\left(\mu_{2}+\frac{1}{2}\right) \cdots \Gamma\left(\mu_{d}+\frac{1}{2}\right) \Gamma\left(s+n+1-\frac{d}{2}\right)}{(m+s) !}
$$

Substituting this into (2.3.3) and further on into (2.3.2) we obtain due to (2.2.2):

$a_{n}(x)=\left.\sum_{m=n}^{4 n} \sum_{k=0}^{m} \frac{(-1)^{k+m-n}}{m !(2 \pi)^{d}}\left(\begin{array}{c}m \\ k\end{array}\right) \Delta^{k} \Delta_{0}^{m-k}\left(\sum_{|\mu|=m-n} \frac{x^{2 \mu}}{(2 \mu) !} \prod_{i=1}^{d} \Gamma\left(\mu_{i}+\frac{1}{2}\right)\right)\right|_{x=0}$

Note that $s$ has cancelled out as one could expect since heat invariants do not depend on $s$ ! 
Now let us simplify this formula. First notice that

$$
\Delta_{0}^{m-k}=(-1)^{m-k} \sum_{|\beta|=m-k} \frac{(m-k) !}{\beta !} \frac{\partial^{2 \beta}}{\partial x^{2 \beta}},
$$

where $\beta=\left(\beta_{1}, \cdots, \beta_{d}\right)$. Using the well-known representation of the $\Gamma$-function

$$
\Gamma(k+1 / 2)=\frac{\sqrt{\pi}(2 k) !}{4^{k} k !}
$$

we also obtain:

$$
\prod_{i=1}^{d} \Gamma\left(\mu_{i}+\frac{1}{2}\right)=\frac{\pi^{d / 2}}{2^{2 m-2 n}} \frac{(2 \mu) !}{\mu !}
$$

Let us substitute (3.1.4) and (3.1.6) into the above formula for the $a_{n}(x)$ and apply $\Delta_{0}^{m-k}$ to $x^{2 \mu}$. Introducing the new summation multiindex $\alpha=\mu-\beta$ and noticing that all terms for $k<n$ vanish we finally obtain

$a_{n}(0)=\left.(4 \pi)^{-\frac{d}{2}}(-1)^{n} \sum_{m=n}^{4 n} \sum_{k=n}^{m} \frac{1}{k ! 2^{2 m-2 n}} \sum_{|\alpha|=m-k} \sum_{|\beta|=k-n} \frac{(2 \alpha+2 \beta) !}{\alpha !(\alpha+\beta) !(2 \beta) !} \Delta^{k}\left(x^{2 \beta}\right)\right|_{x=0}$.

This completes the proof of the theorem.

3.2. Remarks. The proof of the Theorem 3.1.1 is similar to the proofs of main theorems in [P1] and [P2]. One may check that in the particular cases of the 2-dimensional Laplacian and the 1-dimensional Schrödinger operator Theorem 3.1.1 agrees with the results obtained in [P1] and [P2].

\section{INVARIANCE AND COMBINATORIAL IDENTITIES}

4.1. Combinatorial identities. Let us rewrite (3.1.2) in the following way:

$$
\left.(4 \pi)^{-\frac{d}{2}}(-4)^{n} \sum_{k=n}^{4 n} \frac{1}{k !} \Delta^{k}\left(\sum_{m=k}^{4 n} \frac{1}{4^{m}} \sum_{|\beta|=k-n}\left(\sum_{|\alpha|=m-k} \frac{(2 \alpha+2 \beta) ! \beta !}{\alpha !(\alpha+\beta) !(2 \beta) !}\right) \frac{1}{\beta !} x^{2 \beta}\right)\right|_{x=0}
$$

Observe that due to the multinomial theorem

$$
\sum_{|\beta|=k-n} \frac{1}{\beta !} x^{2 \beta}=\frac{1}{(k-n) !}\left(x_{1}^{2}+\cdots+x_{d}^{2}\right)^{k-n} .
$$


Let us recall the following generalization of the binomial coefficients (see $[$ Er]). For real $z \in \mathbb{R}$ and $a \in \mathbb{N}$ set

$$
\begin{aligned}
\left(\begin{array}{l}
z \\
a
\end{array}\right)=\left(\begin{array}{c}
z \\
z-a
\end{array}\right)= & \\
& \frac{\Gamma(z+1)}{\Gamma(a+1) \Gamma(z-a+1)}=\frac{z(z-1) \cdots(z-a+1)}{a !} .
\end{aligned}
$$

We also set $\left(\begin{array}{l}z \\ 0\end{array}\right)=\left(\begin{array}{l}z \\ z\end{array}\right)=1$.

Let us proceed with the following simple combinatorial formula.

\section{Lemma 4.1.3.}

$$
\sum_{a=0}^{u}\left(\begin{array}{c}
z+a \\
a
\end{array}\right)\left(\begin{array}{c}
w+u-a \\
u-a
\end{array}\right)=\left(\begin{array}{c}
z+w+u+1 \\
z+w+1
\end{array}\right)
$$

Proof. Using the method of generating functions (see [Rio]) we have:

$$
\sum_{a=0}\left(\begin{array}{c}
z+a \\
a
\end{array}\right) q^{2 a}=\frac{1}{\left(1-q^{2}\right)^{z+1}}
$$

which implies

$\sum_{u=0}^{\infty} \sum_{a_{1}+a_{2}=u}\left(\begin{array}{c}z+a_{1} \\ a_{1}\end{array}\right)\left(\begin{array}{c}w+a_{2} \\ a_{2}\end{array}\right) q^{2 u}=\frac{1}{\left(1-q^{2}\right)^{z+w+2}}=\sum_{u=0}^{\infty}\left(\begin{array}{c}z+w+u+1 \\ z+w+1\end{array}\right) q^{2 u}$.

This completes the proof of the lemma.

Now we can prove our main combinatorial identity.

Theorem 4.1.5. Let $\alpha, \beta$ be multi-indices of dimension $d$ and let $|\beta|=v$. Then

$$
\sum_{|\alpha|=u} \frac{(2 \alpha+2 \beta) ! \beta !}{\alpha !(\alpha+\beta) !(2 \beta) !}=4^{u}\left(\begin{array}{c}
u+v-1+d / 2 \\
u
\end{array}\right) .
$$

Proof. We proceed by induction over $d$. For $d=1$ we have due to (3.1.5):

$$
\frac{(2 u+2 v) ! v !}{u !(u+v) !(2 v) !}=\frac{4^{u+v} \Gamma(u+v+1 / 2) \sqrt{\pi}}{4^{v} \Gamma(v+1 / 2) u ! \sqrt{\pi}}=4^{u}\left(\begin{array}{c}
u+v-1 / 2 \\
u
\end{array}\right) .
$$

and hence (4.1.6) is valid.

Suppose we have proved the formula (4.1.6) in all dimensions less than some $d>1$. Let us prove it in the dimension $d$. Denote $\alpha_{1}=a$, $\beta_{1}=b$. By induction we may rewrite the sum in (4.1.6) as

$$
\sum_{|\alpha|=u} \frac{(2 \alpha+2 \beta) ! \beta !}{\alpha !(\alpha+\beta) !(2 \beta) !}=\sum_{a=0}^{u} \frac{(2 a+2 b) ! b !}{a !(a+b) !(2 b) !}\left(\begin{array}{c}
u-a-1+l \\
u-a
\end{array}\right) 4^{u-a}
$$


where $l=v-b+\frac{d-1}{2}$. On the other hand,

$$
\frac{1}{4^{a}} \frac{(2 a+2 b) ! b !}{a !(a+b) !(2 b) !}=\left(\begin{array}{c}
a+b-1 / 2 \\
a
\end{array}\right)
$$

and hence (4.1.7) is equal to

$$
4^{u} \sum_{a=0}^{u}\left(\begin{array}{c}
a+b-1 / 2 \\
a
\end{array}\right)\left(\begin{array}{c}
u-a-1+l \\
u-a
\end{array}\right) .
$$

By Lemma 4.1.3 this equals to

$$
4^{u}\left(\begin{array}{c}
u+b+l-1 / 2 \\
u
\end{array}\right)=4^{u}\left(\begin{array}{c}
u+v-1+d / 2 \\
u
\end{array}\right),
$$

which completes the proof of the theorem.

4.2. Proof of Theorem 1.2.1. Set $u=m-k, v=k-n$. Combining Theorem 3.1.1, Theorem 4.1.5 and formula 4.1.1 we obtain the following reformulation of (3.1.2):

$$
\begin{aligned}
& a_{n}(0)= \\
& (4 \pi)^{-d / 2}(-4)^{n} \sum_{k=n}^{4 n}\left(\sum_{m=k}^{4 n}\left(\begin{array}{c}
m+\frac{d}{2}-n-1 \\
m-k
\end{array}\right)\right) \frac{\left.\Delta^{k}\left(|x|^{2 k-2 n}\right)\right|_{x=0}}{k ! 4^{k}},
\end{aligned}
$$

where $|x|^{2}=x_{1}^{2}+\cdots+x_{d}^{2}$. Denote $i=m-k, j=k-n$. By Lemma 4.1.3 the inner sum may be rewritten as

$$
\sum_{i=0}^{4 n-k}\left(\begin{array}{c}
i+d / 2+k-n-1 \\
i
\end{array}\right)=\left(\begin{array}{c}
3 n+d / 2 \\
j+d / 2
\end{array}\right)
$$

Therefore (4.2.1) is equal to

$$
a_{n}(0)=(4 \pi)^{-d / 2}(-1)^{n} \sum_{j=0}^{3 n}\left(\begin{array}{c}
3 n+d / 2 \\
j+d / 2
\end{array}\right) \frac{\left.\Delta^{j+n}\left(|x|^{2 j}\right)\right|_{x=0}}{4^{j} j !(j+n) !}
$$

Consider the function $\rho_{x}(y)^{2}$ which is the square of the distance between the points $x$ and $y$. In normal coordinates centered at the point $x=0$ it is given locally by

$$
\rho_{x}(y)^{2}=\sum_{i, j=1}^{d} g_{i j}(0) y_{i} y_{j}=y_{1}^{2}+\cdots+y_{d}^{2}=|y|^{2}
$$

where $y=\left(y_{1}, . ., y_{d}\right)$ (see $[\mathrm{Du}]$, p. 94). Therefore we may rewrite formula (4.2.2) in an invariant form, namely

$$
a_{n}(x)=(4 \pi)^{-d / 2}(-1)^{n} \sum_{j=0}^{3 n}\left(\begin{array}{c}
3 n+d / 2 \\
j+d / 2
\end{array}\right) \frac{\left.\Delta_{y}^{j+n}\left(\rho_{x}(y)^{2 j}\right)\right|_{y=x}}{4^{j} j !(j+n) !},
$$


where the subscript of the Laplacian means that the operator is acting on functions in $y$-variable. This completes the proof of Theorem 1.2.1.

\section{Application to Computation of the KorteweG-De Vries HIERARCHY}

\subsection{Asymptotics of Schrödinger operator and KdV hierarchy.} In [P2] we have applied the Agmon-Kannai method to computation of the Korteweg-de Vries hierarchy (see [NMPZ]). Let us briefly recall the setting of the problem.

Consider the 1-dimensional Schrödinger operator:

$$
L=\frac{\partial^{2}}{\partial x^{2}}+U(x) .
$$

Its heat kernel $H(t, x, y)$ is the fundamental solution of the heat equation

$$
\left(\frac{\partial}{\partial t}-L\right) f=0
$$

It has the following asymptotic representation on the diagonal as $t \rightarrow$ $0+$ :

$$
H(t, x, x) \sim \frac{1}{\sqrt{4 \pi t}} \sum_{n=0}^{\infty} h_{n}[U] t^{n},
$$

where $h_{n}[U]$ are some polynomials in $U(x)$ and its derivatives.

The KdV hierarchy is defined by (see $[\mathrm{AvSc}])$ :

$$
\frac{\partial U}{\partial t}=\frac{\partial}{\partial x} G_{n}[U]
$$

where

$$
G_{n}[U]=\frac{(2 n) !}{2 \cdot n !} h_{n}[U], \quad n \in \mathbb{N} .
$$

Set $U_{0}=U, U_{n}=\partial^{n} U / \partial x^{n}, n \in \mathbb{N}$, where $U_{n}, n \geq 0$ are formal variables. The sequence of polynomials $G_{n}[U]=G_{n}\left[U_{0}, U_{1}, U_{2}, \ldots\right]$ starts with (see $[\mathrm{AvSc}])$ :

$G_{1}[U]=U_{0}, G_{2}[U]=U_{2}+3 U_{0}^{2}, G_{3}[u]=U_{4}+10 U_{0} U_{2}+5 U_{1}^{2}+10 U_{0}^{3}, \ldots$

In particular, substituting $G_{2}[U]$ into (5.1.1) we obtain the familiar Korteweg-de Vries equation (see [NMPZ]):

$$
\frac{\partial U}{\partial t}=\frac{\partial^{3} U}{\partial x^{3}}+6 U \frac{\partial U}{\partial x}
$$


5.2. Computation of the $\mathbf{K d V}$ hierarchy. In [P2] we have presented explicit formulas for the KdV hierarchy (we refer to [P2] for the history of this question). Theorem 1.2.1 allows to simplify the results of $[\mathrm{P} 2]$.

Theorem 5.2.1. The KdV hierarchy is given by:

$$
G_{n}[U]=\frac{(2 n) !}{2 \cdot n !} \sum_{j=0}^{n}\left(\begin{array}{c}
n+\frac{1}{2} \\
j+\frac{1}{2}
\end{array}\right) \frac{(-1)^{j}}{4^{j} j !(j+n) !} P_{n j}[U]
$$

where the polynomial $P_{n j}[U]$ is obtained from $\left.L^{j+n}\left(x^{2 j}\right)\right|_{x=0}$ by a formal change of variables: $U_{i}(0) \rightarrow U_{i}, i=0, . ., 2 n+2 j-2$.

This expression can be completely expanded due to a formula for the powers of the Schrödinger operator ([Rid]).

Theorem 5.2.2. The polynomials $G_{n}[U], n \in \mathbb{N}$ are equal to:

$$
G_{n}[U]=\frac{(2 n) !}{2 \cdot n !} \sum_{j=0}^{n}\left(\begin{array}{c}
n+\frac{1}{2} \\
j+\frac{1}{2}
\end{array}\right) \frac{(-1)^{j}(2 j) !}{4^{j} j !(j+n) !} \sum_{p=1}^{j+n} \sum_{\substack{k_{1}, \ldots, k_{p} \\
k_{1}+\cdots+k_{p}=2(n-p)}} C_{k_{1}, \ldots, k_{p}} U_{k_{1}} \cdots U_{k_{p}}
$$

where

$$
C_{k_{1}, \ldots, k_{p}}=\sum_{\substack{0 \leq l_{0} \leq l_{1} \leq \cdots \leq l_{p-1}=j+n-p \\
2 l_{i} \geq k_{1}+\cdots+k_{i+1}, i=0, \ldots, p-1 .}}\left(\begin{array}{c}
2 l_{0} \\
k_{1}
\end{array}\right)\left(\begin{array}{c}
2 l_{1}-k_{1} \\
k_{2}
\end{array}\right) \cdots\left(\begin{array}{c}
2 l_{p-1}-k_{1}-\cdots-k_{p-1} \\
k_{p}
\end{array}\right) .
$$

Remark. Formula (5.2.1) was checked using Mathematica ([Wo]) and for $1 \leq n \leq 5$ the results agreed with the already known ones (cf. $[\mathrm{GD}])$.

Acknowledgments. This paper is a part of my Ph.D. research at the Department of Mathematics of the Weizmann Institute of Science. I am very grateful to my Ph.D. advisor Yakar Kannai for his constant help and support.

The author is indebted to Leonid Polterovich, Amitai Regev and Mikhail Solomyak for helpful discussions. I would like to thank Ivan Avramidi, Isaac Chavel, Peter Gilkey and Steven Rosenberg for their remarks on the preliminary versions of this paper. I am also grateful to Sergei Novikov for a useful discussion on the KdV hierarchy and its computation.

Theorem 4.1.5 was first established with the help of a Mathematica implementation of the Wilf-Zeilberger algorithm (see [PWZ]). I am thankful to Marko Petkovšek and Doron Zeilberger for their help with this matter. 


\section{REFERENCES}

[AK] S. Agmon, Y. Kannai, On the asymptotic behavior of spectral functions and resolvent kernels of elliptic operators, Israel J. Math. 5 (1967), 1-30.

[Av] I.G. Avramidi, A covariant technique for the calculation of the one-loop effective action, Nucl. Phys. B 355 (1991) 712-754; Erratum: Nucl. Phys. B 509 (1998) 557-558.

[AvB] I.G. Avramidi, T. Branson, Heat kernel asymptotics of operators with non-Laplace principal part, math-ph/9905001, (1999), 1-43.

[AvSc] I.G. Avramidi and R. Schimming, A new explicit expression for the Korteweg - de Vries hierarchy, solv-int/9710009, (1997), 1-17.

[Be] M. Berger, Geometry of the spectrum, Proc. Symp. Pure Math. 27 (1975), 129-152.

[BGM] M. Berger, P. Gauduchon, E. Mazet, Le spectre d'une variètè Riemannienne, Lecture Notes in Math. 194, Springer-Verlag, 1971.

[BGØ] T. Branson, P. Gilkey, B. Ørsted, Leading terms in the heat invariants, Proc. Am. Math. Soc. 109 (1990), 437-450.

[Ch] I. Chavel, Eigenvalues in Riemannian geometry, Academic Press, 1984.

[Da] E. B. Davies, Heat kernels and spectral theory. Cambridge University Press, 1989.

[Du] G.F.D. Duff, Partial differential equations, University of Toronto Press, 1956.

[Er] A. Erdélyi et. al., Higher transcendental functions, vol. 1, McGrawHill, 1953.

[F] S.A. Fulling ed., Heat Kernel Techniques and Quantum Gravity, Discourses in Math. and its Appl., No. 4, Texas A\&M Univ., 1995.

[GD] I.M. Gelfand, L.A. Dikii, Asymptotic behaviour of the resolvent of Sturm-Liouville equation and the algebra of the Korteweg-de Vries equations, Russian Math. Surveys, 30:5 (1975), 77-113.

[G1] P. Gilkey, The spectral geometry of a Riemannian manifold, J. Diff. Geom. 10 (1975), 601-618.

[G2] P. Gilkey, The index theorem and the heat equation, Math. Lect.Series, Publish or Perish, 1974.

[G3] P. Gilkey, Heat equation asymptotics, Proc. Symp. Pure Math. 54 (1993), 317-326.

[GR] I.S. Gradshtein, I.M. Ryzhik, Table of integrals, series and products, Academic Press, 1980. 
[GKM] D. Gromoll, W. Klingenberg, W. Meyer, Riemannsche Geometrie im Grossen, Springer-Verlag, 1968.

[Hö] L. Hörmander, Linear partial differential operators, Springer-Verlag, 1969.

[MS] H.P. McKean, Jr., I.M. Singer, Curvature and the eigenvalues of the Laplacian, J. Diff. Geom. 1 (1967), 43-69.

[MP] S. Minakshisundaram, A. Pleijel, Some properties of the eigenfunctions of the Laplace-operator on Riemannian manifolds, Canadian J. Math. 1 (1949), 242-256.

[NMPZ] S.P. Novikov, S.V. Manakov, L.P. Pitaevskii, V.E. Zakharov, Theory of solitons: the inverse scattering method, Consultants Bureau, 1984.

[OPS] B. Osgood, R. Phillips, P. Sarnak, Compact isospectral sets of surfaces, J. Funct. Anal. 80 (1988), 212-234.

[PWZ] M. Petkovšek, H. Wilf, D. Zeilberger, A=B, A K Peters, 1996.

[P1] I. Polterovich, A commutator method for computation of heat invariants, to appear in Indagationes Mathematicae, 1-11.

[P2] I. Polterovich, From Agmon-Kannai expansion to Korteweg-de Vries hierarchy, to appear in Letters in Mathematical Physics, 1-7.

[Rid] S.Z. Rida, Explicit formulae for the powers of a Schrödinger-like ordinary differential operator, J. Phys. A: Math. Gen. 31 (1998), 5577-5583.

[Rio] J. Riordan, An introduction to combinatorial analysis, John Wiley \& Sons, Inc., 1958.

[Ro] S. Rosenberg, The Laplacian on a Riemannian manifold, Cambridge University Press, 1997.

[Se] R. Seeley, Complex powers of an elliptic operator, Proc. Symp. Pure Math. 10 (1967), 288-307.

[Sa] T. Sakai, On the eigenvalues of the Laplacian and curvature of Riemannian manifold, Tôhuku Math. J. 23 (1971), 585-603.

[vdV] A.E.M. van de Ven, Index-free heat kernel coefficients, hepth/9708152, (1997), 1-38.

[Wo] S.Wolfram, Mathematica: a system for doing mathematics by computer, Addison - Wesley, 1991.

[Xu] C. Xu, Heat kernels and geometric invariants I, Bull. Sc. Math. 117 (1993), 287-312.

Department of Theoretical Mathematics, The Weizmann Institute of Science, Rehovot 76100, Israel

E-mail address: iossif@wisdom.weizmann.ac.il 